Clinical and demographic characteristics associated with foreign body intake in children aged 4-8 years, cared for at the Children's Hospital of Mexico Federico Gómez over a period of two years

\title{
Características clínicas y demográficas asociadas a la ingesta de cuerpo extraño en niños de 4-8 años de edad, atendidos en el Hospital Infantil de México Federico Gómez en un periodo de dos años
}

Belén Fernández Paxtián

\begin{abstract}
Introduction: accidental intake of foreign bodies (EC) in children is considered a health problem. In 2014 in the United States of America, the American PoisonIng Association Control Center reported that more than 128,000 patients had ingested an EC; more than $69 \%$ had occurred in children under the age of 5 and $83 \%$, in children under the age of 19 .

Objective: to identify and describe the clinical and demographic characteristics associated with the accidental intake of a foreign body by patients aged 4 to 8 years attended at the Children's Hospital of Mexico Federico Gómez and that allows to formulate preventive strategies, applicable in this population. Material and methods: observational retrospective study, records of 75 patients between 4 and 8 years of age were reviewed, of which 65 met the criteria for the study, entered by diagnosis of foreign body intake, treated in HIMFG during the period 2016-2017. Those who had a health problem that conditioned this risk (neurological deterioration) and who were a home dweller were excluded. Central trend and dispersion measures were obtained for quantitative variables (mean, standard deviation, 95\% confidence interval). Relative frequencies of qualitative variables were calculated.

Results: at home is where the event occurs with $91.9 \%$, the accident has the highest incidence during the game, by $80.6 \%$; the intake of solid objects stands out, occupying $91.9 \%$ with coins. Parents are the ones who are at the time of the event and move patients to receive medical care $87.1 \%$ and $51.6 \%$ of these types of accidents. They occurred in non-skilled day; children don't go to school. Friday is considered as a weekend as every last Friday of the month there are no classes.

Conclusion: the game is a factor that does influence this behavior, it is important to direct preventive attention, by applying rules that set limits in the game and not leave them available to children.
\end{abstract}

Keywords: intake, foreign body, coins, accident.

Citación: Fernandez Paxtian Belén. Características clínicas y demográficas asociadas a la ingesta de cuerpo extraño en niños de 4-8 años de edad, atendidos en el Hospital Infantil de México Federico Gómez en un periodo de dos años. Rev Enferm Neurol. 2020;19(2): pp. 66-73

Correspondencia: Fernández Paxtián Belén

Email: maromayou@hotmail.com

EP. Quimioterapia Ambulatoria, Hospital Infantil

de México Federico Gómez
Recibido: 17 de febrero 2020

Aceptado: 23 de marzo 2020

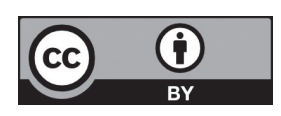




\section{Resumen}

Introducción: la ingesta accidental de cuerpos extraños (CE) en niños, es considerada un problema de salud. En el 2014 en Estados Unidos de Norteamérica, el Centro de Control de la Asociación Americana de Envenenamientos reportó que más de 128000 pacientes habían ingerido un CE; más del $69 \%$ había ocurrido en menores de 5 años y 83\%, en menores de 19 años.

Objetivo: identificar y describir las características clínicas y demográficas asociadas con la ingesta accidental de un cuerpo extraño por pacientes de 4 a 8 años edad atendidos en el Hospital Infantil de México Federico Gómez, que permita formular estrategias preventivas, aplicables en esta población. Material y métodos: estudio retrospectivo observacional, se revisaron expedientes de 75 pacientes entre los 4 a 8 años de edad, de los cuales 65 reunieron los criterios para el estudio, ingresados por diagnóstico de ingesta de cuerpo extraño, tratados en el HIMFG durante el periodo de 2016-2017. Se excluyeron quienes presentaron un problema de salud que condicionara este riesgo (deterioro neurológico) y que fuera habitante de casa hogar. Se obtuvieron medidas de tendencia central y dispersión para las variables cuantitativas (media, desviación estándar, intervalo de confianza de 95\%). Se calcularon frecuencias relativas de las variables cualitativas.

Resultados: en el hogar es donde ocurre el evento con un 91.9\%, el accidente tiene mayor incidencia durante el juego, en un 80.6\%; sobresale la ingesta de objetos sólidos, ocupando el $91.9 \%$ con las monedas. Los padres son quienes se encuentran en el momento del evento y trasladan a los pacientes a recibir atención médica $87.1 \%$ y el $51.6 \%$ de este tipo de accidentes. Se produjeron en día no hábil; los niños no acuden a la escuela. Se considera el viernes como fin de semana pues cada último viernes del mes no hay clases.

Conclusión: el juego es un factor que sí influye en este comportamiento, es importante dirigir la atención preventiva, mediante la aplicación de reglas que establezcan límites en el juego y no dejarlas al alcance de los niños.

Palabras clave: ingesta, cuerpo extraño, monedas, accidente.

\section{Introducción}

Los niños en etapa preescolar y escolar se caracterizan por el interés en explorar las situaciones y los elementos que se encuentren en su entorno. Esa curiosidad los hace más vulnerables para presentar accidentes y exponer varios elementos como factores de peligro en su convivencia diaria.

Cuerpo extraño proviene del latín ekstajno y se define como algo ajeno a la naturaleza de algo. Es cualquier elemento ajeno al cuerpo que entra a este a través de la piel o por cualquier orificio natural como ojos, nariz, garganta y que a su vez impide su funcionamiento normal.

La ingestión de CE es un accidente muy común en la infancia, ocupa el segundo lugar como motivo para la realización de broncoscopía. Por su importancia y frecuencia puede considerarse como un problema de salud pública. Es preponderante, detectar factores de riesgo que favorece la incidencia de este problema para prevenir el suceso y evitar la exposición de un niño sano a una serie de procedimientos invasivos. 
Rikke Haarhr Iversen, Tejs Ehlers Klug, 2012, en su artículo titulado: "Necesidad de recomendaciones claras a los padres respecto a la aspiración de cuerpos extraños en el niño”, publicada en Dan Med J, realiza un estudio retrospectivo en el que revisa archivos de pacientes de 0-15 años de edad quienes fueron ingresados con sospecha de ingesta de cuerpos extraños y confirmados a través de la endoscopia. De 136 casos sometidos a broncoscopía la mediana que arrojo la edad promedio fue de 1 año y de ellos 59 presentaron un cuerpo extraño de los que el $86 \%$ era material orgánico y el $14 \%$ inorgánico.

Menciona factores importantes, como: la dentición incompleta, coordinación inmadura de la deglución y tendencia a la distracción durante la alimentación.

Concluye que la simple sospecha de la ingestión de cuerpo extraño conlleva a la práctica de una broncoscopía, que a su vez es importante su realización dentro de las primeras 24 hs para reducir riesgos de complicaciones por el accidente.

Por último, toca la importancia de la concientización de los peligros asociados a la alimentación en niños menores de 3 años.

A pesar de que el estudio se enfoca a la edad pediátrica sólo abarca los primeros años de vida, y en el Hospital Infantil de México Federico Gómez, el rango de edad tiende a variar hacia la edad preescolar y escolar, lo que origina diferir en los factores de riesgo mencionados por el autor; sin embargo, no deja de tener importancia la participación de las personas que se encuentren al cuidado de los menores, así como determinar causalidad para prevención.

\section{Material y métodos}

Es un estudio descriptivo, retrospectivo, observacional; se revisaron expedientes de pacientes ingresados con diagnóstico de ingesta de $\mathrm{CE}$, que hayan sido tratados en el HIMFG, en el periodo de 2016-2017. Pacientes entre los 4 a 8 años de edad, de 75 expedientes registrados, sólo 65 cumplieron con los estándares del estudio, se excluyeron 3 casos por presentar un problema de salud que condicionara este riesgo (deterioro neurológico) y habitante de casa hogar. Las variables dependientes cualitativas: estado de salud, lugar del suceso; independientes: día del evento, tipo de objeto ingerido, actividad realizada durante el suceso y nivel socioeconómico (basado en el nivel establecido por el tabulador hospitalario). Y la variable dependiente cuantitativa: la edad. Análisis de los datos: se obtuvieron medidas de tendencia central y dispersión para las variables cuantitativas (media, desviación estándar, intervalo de confianza 95\%. Se calcularon frecuencias relativas de las variables cualitativas)

\section{Resultados}

Del total de pacientes incluidos la edad promedio 70 semanas $(5 \mathrm{a} 10 \mathrm{~m})$ con predominio del sexo femenino 63\%. El lugar más frecuente donde ocurre el evento el hogar con $91.9 \%$ en torno a esta situación el accidente tiene mayor relevancia durante el juego $80.6 \%$; de la misma manera sobresale la ingesta de objetos sólidos $91.9 \%$ con las monedas. Los padres son quienes se encuentran en el momento del evento y trasladan a los pacientes a recibir atención médica $87.1 \%$; en un $51.6 \%$ de estos accidentes, se llevaron a cabo en día no hábil, en que los niños no acuden a la escuela, en el estudio la eventualidad se presentó en fin de semana, debido a que cada viernes último de mes no hay clases, con base en el calendario escolar de la SEP ( Secretaría de Educación Pública) que lo asigna para reuniones técnicas de los profesores. Antes de esto, la condición de los niños, se registra como sana. 
Tabla 1. Edad promedio

\begin{tabular}{ccc}
\hline Variable & Media & Desv. Estánda \\
\hline Edad (meses) & 70.01 & 16.19 \\
\hline
\end{tabular}

Fuente: Hospital Infantil de México Federico Gómez

Elaboró: Belén Fernández Paxtián

Gráfica I. Niños (as) con ingesta de cuerpo extraño $n=62$

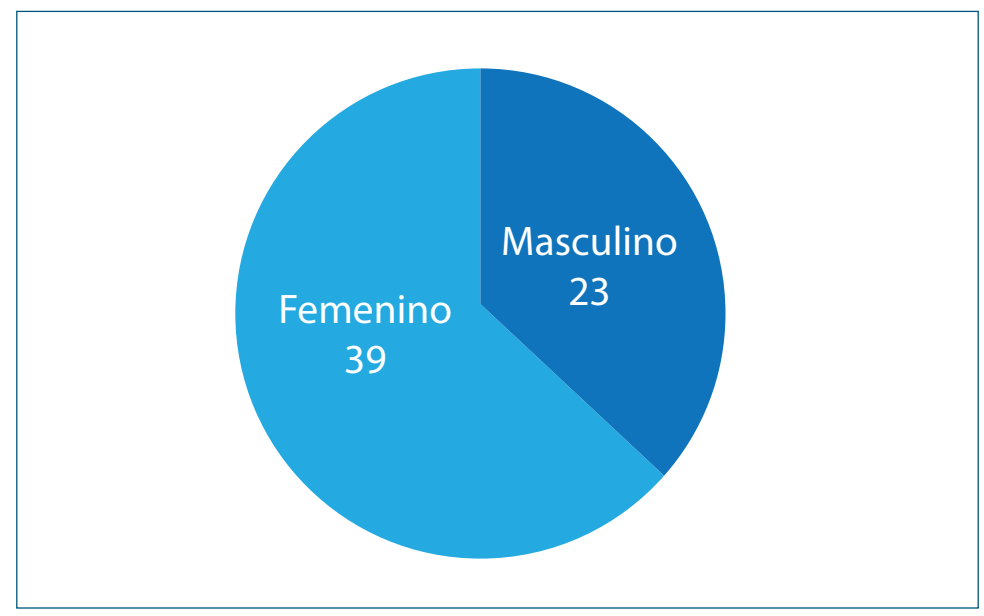

Fuente: Hospital Infantil de México Federico Gómez

Elaboró: Belén Fernández Paxtián

Gráfica II. Etapa de desarrollo de los sujetos de estudio $\quad n=62$

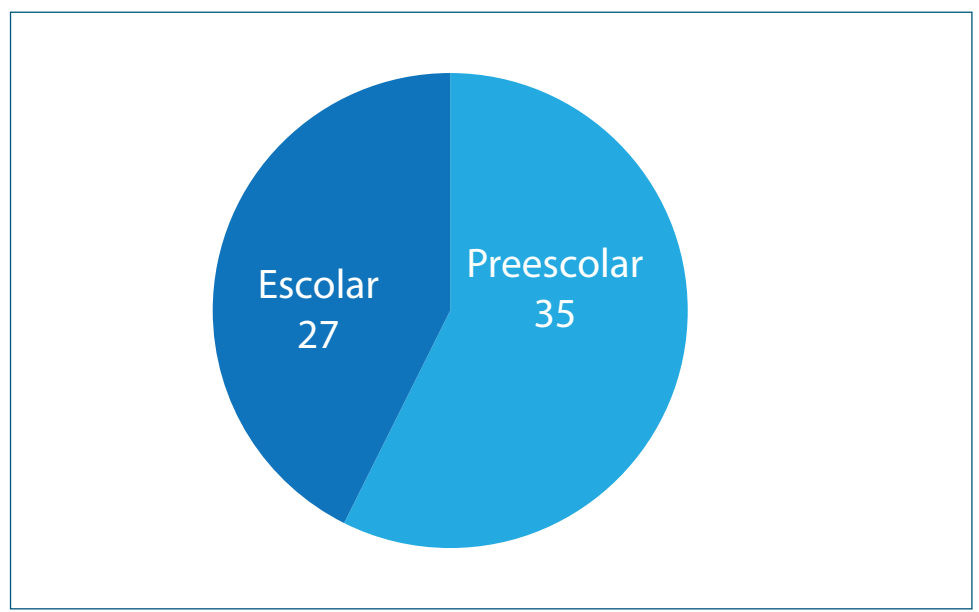

Fuente: Hospital Infantil de México Federico Gómez

Elaboró: Belén Fernández Paxtián 


\section{Gráfica III. Lugar donde ocurrió el evento $\quad$ n=62}

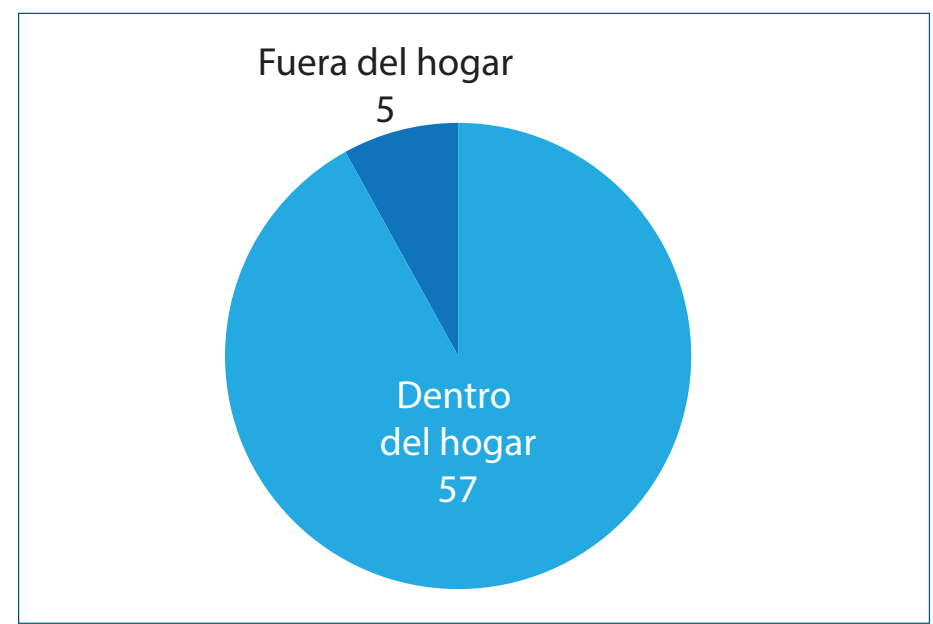

Fuente: Hospital Infantil de México Federico Gómez

Elaboró: Belén Fernández Paxtián

Gráfica IV. Actividad previa a la ingesta del cuerpo extraño $\quad n=62$

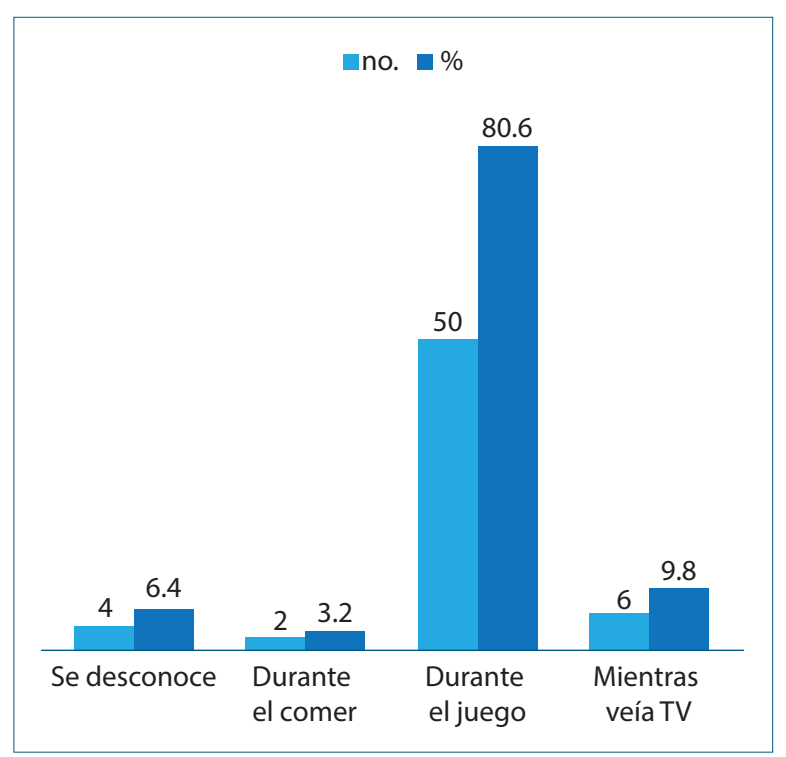

Fuente: Hospital Infantil de México Federico Gómez Elaboró: Belén Fernández Paxtián 
Gráfica V. Tipo de material ingerido por los niños de estudio $n=62$

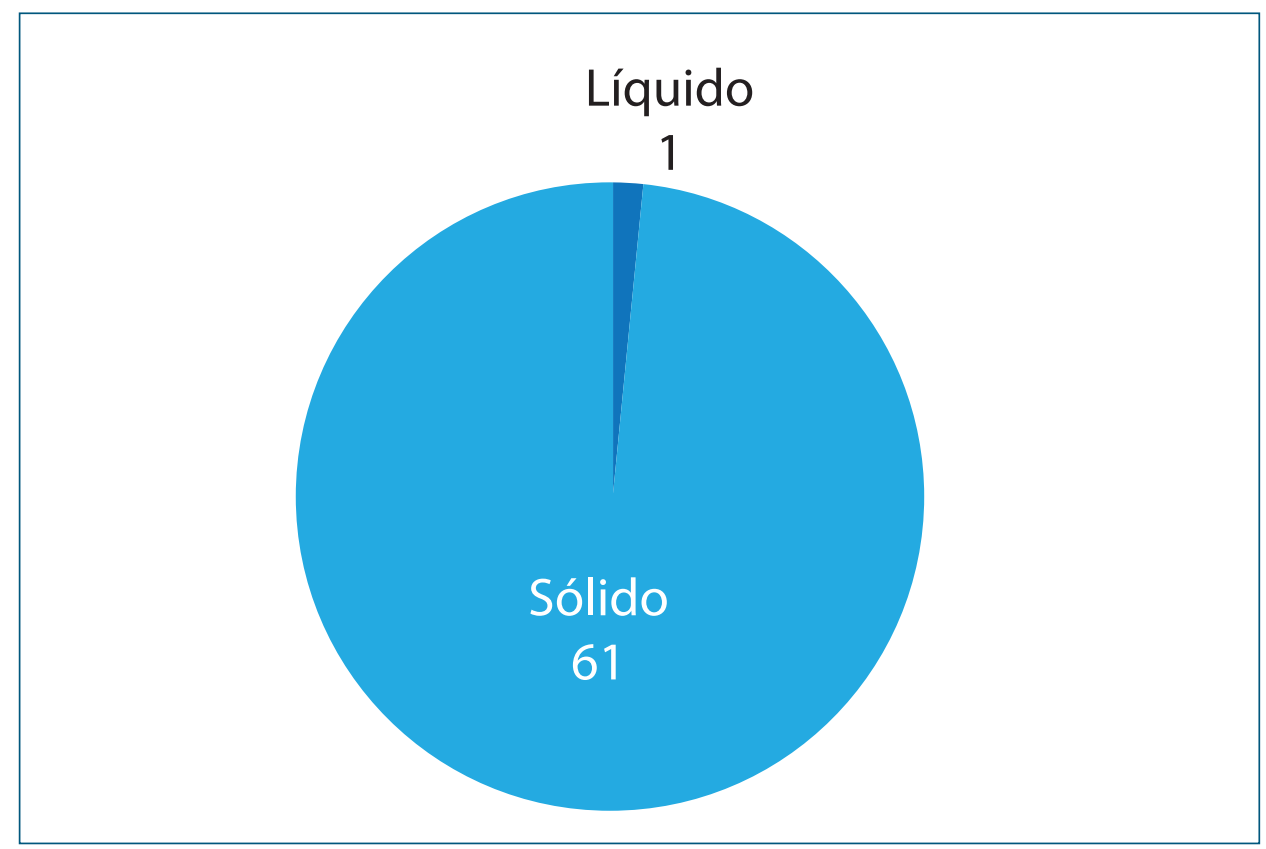

Fuente: Hospital Infantil de México Federico Gómez

Elaboró: Belén Fernández Paxtián

Gráfica VI. Material ingerido por los niños del estudio $n=62$

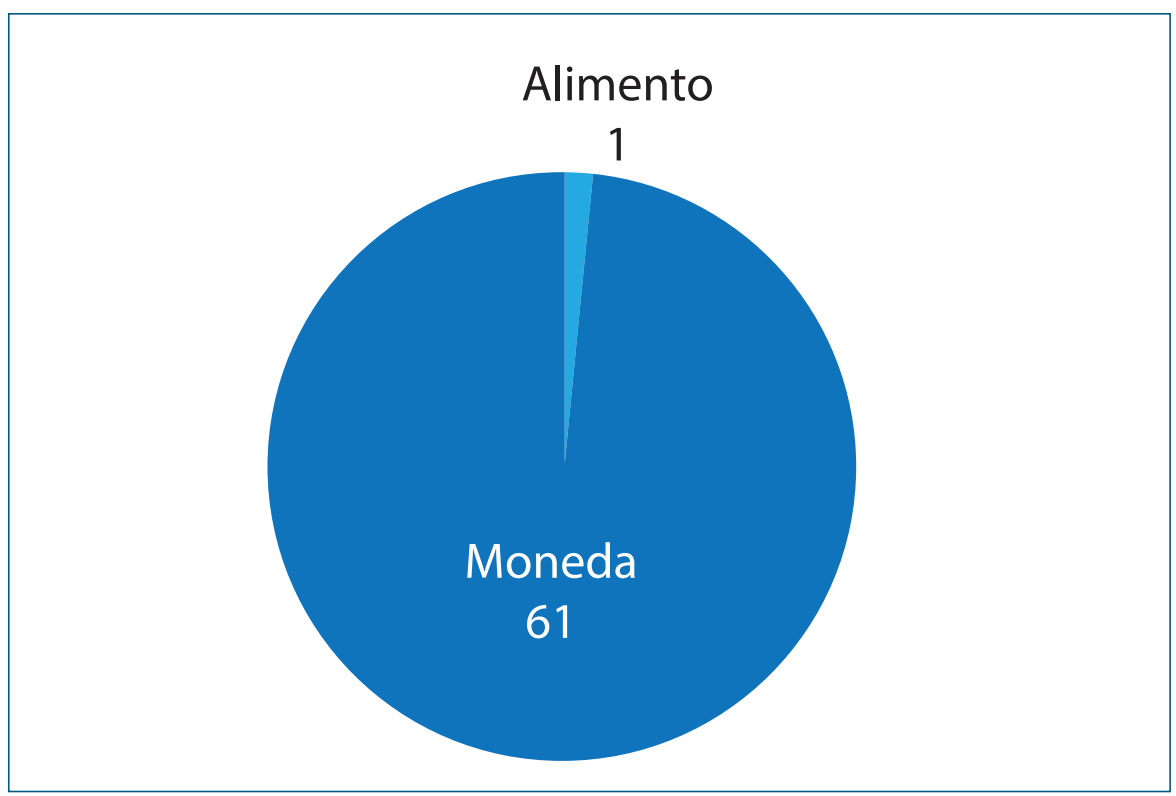

Fuente: Hospital Infantil de México Federico Gómez

Elaboró: Belén Fernández Paxtián 
Gráfica VII. Persona presente en el momento de la ingesta del cuerpo extraño $n=62$

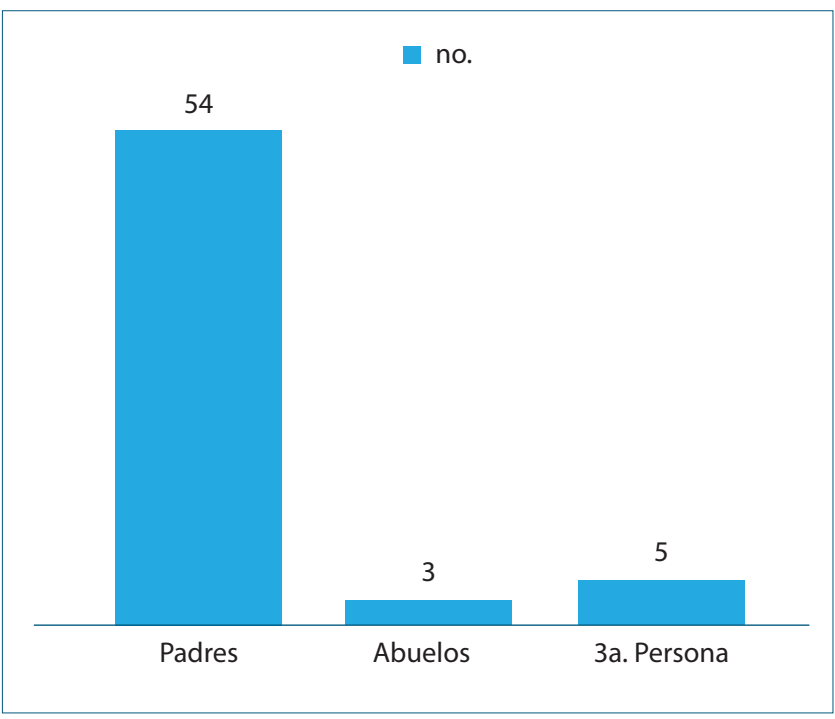

Fuente: Hospital Infantil de México Federico Gómez Elaboró: Belén Fernández Paxtián

\section{Discusión}

En relación con lo reportado por Rikke Haarhr Iversen, Tejs Ehlers Klug en su artículo, los niños estudiados tienen una edad promedio de un año, en su mayoría el material fue orgánico, los niños no tenían dentición completa e inmadurez en su deglución y coordinación, además de tendencia a la distracción durante la alimentación. En el presente estudio el material ingerido fue inorgánico, la diferencia en edad, en éste es que son mayores de 3 años, en consecuencia, se cree que ya se tiene mayor conocimiento del peligro; sin embargo, el accidente se manifiesta en su mayoría durante un evento de juego, por esta razón, pensamos que es producto de un descuido o distracción por parte del niño y a su vez de la persona con quien se encuentra, la incidencia predomina en día no hábil. Por ello, es importante fomentar las medidas preventivas en el hogar dirigidas a evitar acciden- tes, es vital que la comunidad conozca y aprenda la aplicación de técnicas básicas ante este tipo de acontecimientos; asimismo, estén informados de los riesgos que implica el someter a un niño a una endoscopia tanto por la anestesia como por el mismo procedimiento.

Blanco-Rodríguez G, et al., en su investigación predominó el sexo masculino (50,9\%); $74 \%$ fueron menores de 5 años. El estudio utilizado fue la radiografía que permitió localizar el CE al igual que, en nuestro estudio la moneda $(78,3 \%)$ el más ingerido.

En cuanto al sexo, el femenino supera al masculino quizás por la relación mujer: hombre, por el desarrollo psicomotor evolutivo etapa de la creatividad en que los niños en general a todo le dan vida y nombre, gracias a su pensamiento mágico, en que nada es imposible y se atreven a realizar y probar cosas que tiene a su alcance.

\section{Conclusión}

De lo precedente, vemos como la inquietud de un niño supera las expectativas de todo padre o cuidador inmediato, los niños en edad preescolar y escolar, aún requieren mayor vigilancia ante la ingesta de CE, debido a que están muy ocupados con el juego; en consecuencia, los cuidadores deben estar enterados de los riesgos que presentan los niños en esta edad como parte de la prevención. En relación con el sexo, se identificó que el femenino es quien presenta mayor riesgo, con certeza habrá factores evolutivos que impliquen nuevos estándares.

La investigación permite afirmar que el juego o distracción, sí influye en la ingesta de CE, la mayoría fueron monedas; se recomienda no dejarlas al alcance de los niños, puesto que no se puede 
eliminar su uso. De igual manera, se deben establecer reglas a los niños, para evitar y prevenir accidentes; pues va en incremento, es importante dirigir la atención al cuidado, lo expuesto, comprueba la existencia de un problema de salud.

\section{Referencias}

1. Rikke HI, Tejs EK. Need for more clear parental recommendations regarding foreign body aspiration in children. Dan Med J 2012;59(9) A4498:1-4.

2. Blanco-Rodríguez G, Teyssier-Morales G, Penchyna-Grub J, Madriñan-Rivas E, RivasRivera IA, Trujillo-Ponce de León A, et al. Características y resultados de la ingestión de cuerpos extraños en niños. Arch Argent Pediatr 2018;116(4):256-61.

2. Frederick PR. where do we go from here? Pediatric Injury Control. Pediatrics supl 1999;83-8.

3. Predes SRS. Boletín Médico del Hospital Infantil de México. 2000;57(7):375.

4. Sánchez GS, Martín AA. Cuerpos extraños traqueobronquiales en la infancia. Estudio de 133 casos y revisión de la literatura. Acta Otorrinol Esp 1990;41:309-16.

5. Aytac A, Ikizler C, Olga R, Sayleim A. Inhalation of foreign bodies in children. Report of 500 cases. J Thorac Cardiovasc Surg 1977;74:145-51.

6. Fernández I, Gutiérrez C, Álvarez V, Peláez D. Broncoaspiración de cuerpos extraños en la infancia. Revisión de 210 casos. Ann Esp Pediatr 2000;53:335-8.

7. Schol MY, Prego J, de Leonardis D. Aspiración de cuerpos extraños en niños. Arch Pediatr Urug 1999;70:53-64.

8. Gans SL, Berci G. Advances in endoscopy of infants and children. J Pediatr Surg 1971;6:199-233.

9. Lobeiras Ana, Sugazabeitia Amaia, Uribarri Nerea, Mintegi Santiago. Consultas relacionadas con ingesta de un cuerpo extraño en urgencias. An Pediatr (Barc) 2017;86(4):182-7.
10. Cadena León José F, Cázares-Méndez Josefina M, Toro Monjaraz Erick M, Cervantes Bustamente Roberto, Ramírez-Mayans Jaime. Manejo de la ingesta de cuerpos extraños por vía digestiva. Acta Pediatr Mex 2019;40(5):290-4. 